\title{
Anatabine Attenuates Tau Phosphorylation and Oligomerization in P301S Tau Transgenic Mice
}

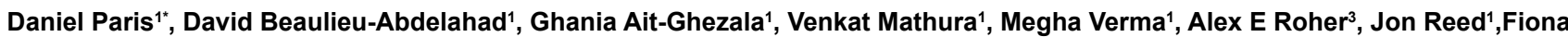

Crawford $^{1}$ and Michael Mullan ${ }^{1,2}$

${ }^{1}$ Roskamp Institute, Sarasota, Florida, USA

${ }^{2}$ Rock Creek Pharmaceuticals, Gloucester, Massachusetts, USA

${ }^{3}$ The Longtine Center for Neurodegenerative Biochemistry, Banner Sun Health Research Institute, Sun City, Arizona, USA

\begin{abstract}
We have previously shown that the natural alkaloid anatabine displays some anti-inflammatory and Alzheimer amyloid (A $\beta$ ) lowering properties in the central nervous system associated with reduced STAT3 and NFkB activation. We investigated here the impact of a chronic oral treatment with anatabine in a model of tauopathy. We found that anatabine reduces the incidence of paralysis and abnormal hind limb extension reflex while improving rotarod performances in P301S mutant human Tau transgenic mice ( $\mathrm{Tg}$ Tau P301S) suggesting that anatabine delays the disease progression in this model of tauopathy. Analyzes of brain and spinal cord homogenates reveal that anatabine reduces tau phosphorylation at multiple pertinent Alzheimer's disease (AD) epitopes and decreases the levels of pathological tau conformers/oligomers in both detergent soluble and insoluble fractions. Pathological tau species reduction induced by anatabine was accompanied by decreased lba1 expression suggesting a diminution of microgliosis in the brain and spinal cord of Tg Tau P301S mice. In addition, we found that anatabine administration increases phosphorylation of the inhibitory residue (Ser9) of glycogen synthase kinase-3 $\beta$, a primary tau kinase, associated with AD pathology, providing a possible mechanism for the observed reduction of tau phosphorylation. These data support further exploration of anatabine as a possible disease modifying agent for neurodegenerative tauopathies and, in particular $A D$, since anatabine also displays $A \beta$ lowering properties.
\end{abstract}

\section{Keywords}

Tauopathy; Alzheimer; Tau; Phosphorylation; oligomer; Microgliosis; Anatabine; Glycogen synthase kinase; Protein kinase B; AKT

\section{Introduction}

Alzheimer's disease (AD), the most prevalent form of dementia in the elderly, is a progressive neurodegenerative disorder characterized by a loss of cognitive functions and by diverse psychiatric symptoms leading to confusion, agitation and aggression which constitute a major cause of patient distress. $\mathrm{AD}$ pathology is characterized by the deposition of extracellular $A \beta$ peptides and by intraneuronal accumulation of hyperphosphorylated and aggregated tau protein [1]. Beside the pathological accumulation of intraneuronal neurofibrillary tangles and extracellular $\mathrm{A} \beta$ deposits, neurodegeneration and neuroinflammation associated with microgliosis and astrogliosis are prevalent in $\mathrm{AD}$ brains. $\mathrm{AD}$ neurodegeneration is widespread and affects many brain areas. Cholinergic dysfunction in the basal and rostral forebrain correlates with early cognitive dysfunctions in $\mathrm{AD}$ and is well documented [2]. In addition to the loss of cholinergic neurons, there is also a significant loss of noradrenergic neurons in the locus coeruleus (LC) which may contribute to cognitive impairment and depression in $\mathrm{AD}$ [3]. Norepinephrine mainly originates from the LC in the central nervous system and exerts an anti-inflammatory activity via activation of glial adrenoreceptors (AR), therefore LC neurodegeneration may also contribute to neuroinflammation in $\mathrm{AD}$ [4]. AR have also been implicated in $A \beta$ formation and post-receptor components including the $\mathrm{G}$ protein coupled receptor kinase 2 (GRK2) (responsible for the desensitization of $\mathrm{AR}$ ) is altered by $\mathrm{A} \beta$ and may also mediate $A \beta$ induced AR dysfunction in neurons [4]. Moreover, GRK2 has been shown to result in tau hyperphosphorylation and is colocalized with neurofibrillary tangles in $\mathrm{AD}$ [5] suggesting that it may also contribute to tau pathology in $\mathrm{AD}$ highlighting $\mathrm{AR}$ signaling and GRK2 modulation as valuable therapeutic targets for AD [4].
The transcription factors STAT3 and NFkB are activated in AD brains and play a key role in $\mathrm{AD}$ neuroinflammation by driving the expression of a large array of pro-inflammatory genes including cytokines, cyclooxygenase- 2 and the inducible nitric oxide synthase $[6,7]$. Interestingly, $A \beta$ has been shown to stimulate STAT3 phosphorylation resulting in microglial activation [8] and neuronal death $[6,9]$ further highlighting the importance of STAT3 in the pathophysiology of $\mathrm{AD}$. Rare familial forms of $\mathrm{AD}$ are caused by mutations in the amyloid precursor protein (APP) or presenilin genes resulting in an increased production and accumulation of $A \beta$ peptides [10]. $A \beta$ peptides have therefore been considered as a major target for $\mathrm{AD}$ drug discovery. However, to date, $A \beta$ targeted therapies have failed to show efficacy in late stage clinical trials suggesting that $A \beta$ may not be a sufficient target for $\mathrm{AD}$ therapeutic development. Pathological tau aggregation and accumulation is highly correlated with AD disease progression whereas a poor correlation has been observed between $\mathrm{AD}$ and $\mathrm{A} \beta$ deposition $[11,12]$ suggesting that tau could represent an alternative target for $\mathrm{AD}$ drug development.

Although no mutation in the tau gene have been linked to AD, several mutations causing frontotemporal dementia and parkinsonism have been indentified [13] showing that abnormal forms of tau can directly cause neurodegeneration and memory loss. Also, a tau haplotype responsible for higher tau expression [14] and GAB2

*Corresponding author: Daniel Paris, Roskamp Institute, Sarasota, Florida, USA, Tel: 941-752-2949; Fax: 941-752-2948; E-mail: dparis@rfdn.org

Received April 21, 2014; Accepted May 21, 2014; Published May 21, 2014

Citation: Paris D, Beaulieu-Abdelahad D, Ait-Ghezala G, Mathura V, Verma M, et al, (2014) Anatabine Attenuates Tau Phosphorylation and Oligomerization in P301S Tau Transgenic Mice. Brain Disord Ther 3:126. doi:10.4172/2168-975X.1000126

Copyright: ( 2013 Paris D, et al. This is an open-access article distributed under the terms of the Creative Commons Attribution License, which permits unrestricted use, distribution, and reproduction in any medium, provided the original author and source are credited. 
alleles leading to increased tau phosphorylation appear to augment $\mathrm{AD}$ risk [15] suggesting an influential role of tau in $\mathrm{AD}$. Abnormal tau phosphorylation in $\mathrm{AD}$ is believed to induce tau dissociation from microtubules, allowing tau to oligomerize and aggregate into neurofibrillary tangles, resulting in neurodegeneration. Multiple kinases responsible for the phosphorylation of tau at $\mathrm{AD}$-pertinent epitopes have been identified and could constitute attractive targets for $\mathrm{AD}$ [16]. In particular, glycogen synthase kinase 3 (GSK3) is an ubiquitously-expressed serine/threonine kinase which phosphorylates tau at most serine and threonine residues in hyperphosphorylated neurofibrillary tangles, also contributes to $A \beta$ production [17], and is therefore considered to be a prime target for the treatment of AD.

Several lines of transgenic mice overexpressing human tau mutations have been developed to mimic neurodegenerative tauopathies. These animal models have revealed that soluble tau oligomers rather than larger insoluble tau aggregates present in neurofilbrillary tangles are responsible for behavioral deficits and neurodegeneration [18,19]. In particular, mice overexpressing the human tau gene with the P301S mutation (Tg Tau P301S) have been produced [20]. These mice develop motor impairments, synaptic deficits, microglial activation and neurodegeneration before tangle formation [20] and constitute an interesting model to investigate tau hyperphosphorylation and oligomerization in the brain and the spinal cord.

We have previously identified anatabine, a natural alkaloid present in plants of the Solanacea family, as a novel anti-inflammatory compound antagonizing both STAT3 and NFkB activation [2123]. We have shown that anatabine lowers $A \beta$ production and neuroinflammation both in vitro and in vivo suggesting it could be a useful therapeutic compound against $\mathrm{AD}$, particularly as it readily crosses the blood-brain barrier [21-23]. Other studies have revealed that anatabine suppresses the induction of cycloxygenase- 2 and inducible nitric oxide synthase in macrophage and reduces the incidence of tyroiditis in mice [24] further supporting an anti-inflammatory activity of anatabine in vivo. A randomized controlled clinical trial has shown that anatabine supplementation can reduce thyroglobulin antibodies in patients with Hashimoto's thyroiditis [25] whereas a survey of people using a dietary supplementation of anatabine has shown a beneficial effect on joint pain and stiffness [26] suggesting that anatabine exerts an anti-inflammatory activity also in humans. We investigated here the effect of anatabine on tau phosphorylation and oligomerization in a pure tauopathy model (Tg Tau P301S) [20]. Our data show that anatabine reduces motor impairments as well as tau phosphorylation and oligomerization in the brain and the spinal cord of Tg Tau P301S mice suggesting that anatabine should be further explored for the treatment of tauopathies and $\mathrm{AD}$ in particular.

\section{Materials and Methods}

\section{Mice}

All the experimentations involving mice were approved by the Institutional Animal Care and Use Committee of the Roskamp Institute (IACUC protocol \#44) and were performed in the Association for Assessment and Accreditation of Laboratory Animal Care International (AAALAC) accredited vivarium of the Roskamp Institute. Tg Tau P301S mice (line PS19) [20] were purchased from the Jackson laboratory (ME, USA). Animals were singly housed in a ventilated rack during the duration of the study. Initially, $12 \mathrm{Tg}$ Tau P301S were utilized in the placebo group and $11 \mathrm{Tg}$ Tau P301S were included in the anatabine treatment group. Anatabine was provided by Rock Creek Pharmaceuticals (MA, USA). Mice were treated for 40 days with anatabine at a dosage of $20 \mathrm{mg} / \mathrm{Kg}$ of body weight per 24 hours supplemented in their drinking water or received regular drinking water (placebo group) as previously described [23]. The anatabine treatment was initiated in 28 week-old Tg Tau P301S mice. During the duration of the treatment (40 days), $3 \mathrm{Tg}$ Tau P301S mice in the placebo group became paralyzed and one of them died prematurely before the completion of the study and was not included for the biochemical analyzes of the brain and spinal cord. None of the mice showing paralysis were included in the behavioral analyzes. Animals were humanely euthanatized after 40 days of anatabine treatment and their brains (without cerebellum) and spinal cord were snap frozen in liquid nitrogen for biochemical analyzes.

\section{Hind-limb extension test}

After 39 days of treatment with anatabine, Tg Tau P301S mice were suspended by the tail and the degree of hind-limb extension was observed during 15 seconds. Animals showing an extension reflex in both hind-limbs were considered normal whereas mice displaying an extension reflex in only one hind-limb with partial retraction of the other hind-limb or an absence of extension reflex in both hind-limb (clasping behavior) were considered to have an abnormal extension reflex as previously described [20,27].

\section{Accelerating rotarod test}

Motor coordination was assessed with an accelerating rotarod apparatus (the apparatus was set to accelerating mode from 5 to 40 $\mathrm{rpm}$ in 60 seconds). On day 39 of the anatabine treatment, mice were trained on the rotarod and it was verified that each mouse was able to maintain its balance on the rod. Approximately one hour after this training session, mice were tested on the accelerating rotarod and the latency until a fall occurred recorded for each mouse.

\section{Elevated Plus Maze}

Anxious behavior was assessed using an elevated plus maze composed of two opposite open arms and two opposite closed arms separated by a central platform at $50 \mathrm{~cm}$ above the floor as previously described [28]. Animals were placed in the center of the maze facing an open arm and were allowed to explore the maze for 10 minutes. The time spent by the animals in the different arms of the maze was quantified using the EthoVision XT 8.0 video tracking software (Noldus Information Technology). Anxious behavior was assessed in Tg Tau P301S after 21 days of treatment with anatabine.

\section{Dot blot analyzes}

Mice (7.8 month-old) were humanely euthanatized after 40 days of treatment with anatabine and their brain and spinal cord were dissected out and snap frozen in liquid nitrogen. Brain (without cerebellum) and spinal cord samples from Tg Tau P301S mice were homogenized in 4 volumes of ice cold M-PER reagent (Pierce Biotechnology, IL, USA) containing 1X Halt protease and phosphatase inhibitor cocktail (Thermo Scientific, IL, USA). Samples were centrifuged at 20,000 g for 30 minutes at $4^{\circ} \mathrm{C}$, the supernanatant was collected (M-PER soluble) and the insoluble pellet was resuspended in 2 volumes of MPER buffer containing proteases and phosphatases inhibitor by vigorous vortexing. $5 \mu \mathrm{l}$ of each sample was spotted directly onto nitrocellulose membranes (Biorad, CA, USA). Membranes were blocked for 1 hour at room temperature in 5\% non-fat dry milk dissolved in Tris buffer saline (Biorad, CA, USA) and incubated with the primary antibodies overnight at $4^{\circ} \mathrm{C}$. A different nitrocellulose membrane was used for each of the primary antibody tested. Membranes were hybridized with 
the antibodies CP13 (pSer202) (1/1000 dilution), PHF-1(pSer396/ Ser404) (1/1000 dilution), RZ-3 (pThr231) (1/1000 dilution) to quantify tau phosphorylation at multiple AD relevant epitopes [29]. Pathological tau conformers and oligomers were detected using the conformational antibodies MC1 (1/1000 dilution) [29-31] and TOC1 (1/5000 dilution) [32-33]. Microgliosis was detected with the antibody Iba1 (1/1000 dilution) (Millipore Corporation, MA, USA). After the hybridization, membranes were rinsed in distilled water for 30 minutes and incubated for 2 hours at room temperature with secondary antimouse or anti-rabbit antibodies conjugated with peroxidase (Cell Signaling technology, MA, USA). Signal detection and quantification were performed with a 2 fold dilution of the SuperSignal west fento maximum sensitivity chemiluminescent substrate (Thermo Scientific, IL, USA) by chemiluminescence imaging with the ChemiDocTM XRS (Bio-Rad, CA, USA). After hybridization with the primary antibodies, all membranes were stripped for one hour at room temperature with the RestoreTM western blot stripping buffer (Thermo Scientific, IL, USA) and blocked with 5\% non-fat dry milk. Membranes were then hybridized overnight at $4^{\circ} \mathrm{C}$ with a $1 / 1000$ dilution of a GAPDHHRP conjugated antibody (Santacruz, CA, USA) which was used as a reference antibody to adjust for variation in protein amounts spotted onto nitrocellulose membranes. All results were expressed as a ratio of test antibody/GAPDH chemoluminescent signals.

\section{Human brain samples}

For validation studies 10 non-demented (ND) control individuals (average age 80.7 years; range: $70-96$ years) and $14 \mathrm{AD}$ subjects (average age 80.3 years; range: 61-91 years) were selected. Brain samples were obtained from The Brain Donation Program at Banner Sun Health Research Institute. The operations of the Brain Donation Program and therefore the Neuropathology Core, including the autopsy consent form, have been subjected to and have been approved by the Institutional Review Board of Sun Health Corporation. Donors have all volunteered specifically for the program and all enrolled subjects or legal representatives have signed an Institutional Review Boardapproved informed consent form.

All specimens had short postmortem delay times (NDC mean $3.6 \mathrm{~h}$ and $\mathrm{AD} 2.5 \mathrm{~h}$ ). All AD cases fulfilled Consortium to Establish a Registry for $\mathrm{AD}$ (CERAD criteria) for definite and probable AD. One-hundred $\mathrm{mg}$ of gray matter from the frontal cortex was homogenized in $1 \mathrm{ml}$ of RIPA buffer (Sigma, St. Louis, MO, USA) containing a protease inhibitor cocktail (Roche Diagnostics, Mannheim, Germany) with an Omni TH electric grinder (Kennesaw, GA, USA). The homogenates were centrifuged for $20 \mathrm{~min}$ at 14,000 x g (Beckman 22R centrifuge, Fullerton, CA, USA) and the supernatants collected for analysis. Tau phosphorylation and tau oligomerization were assessed by dot-blots as described above.

\section{SDS-PAGE and Western Blot Analyzes}

The expression of total tau, GSK3 $\beta$ (Ser9) phosphorylation and AKT (Thr308) phosphorylation were determined using SDS-polyacrylamide gel electrophoresis (PAGE) coupled with Western blot analysis of brain and spinal cord homogenates that were dissolved in Laemli buffer (Biorad, CA, USA) containing $\beta$-mercaptoethanol and boiled for 15 minutes. For the detection of tau oligomers by Western blot, brain and spinal cord homogenates were dissolved in Laemli buffer that did not contain $\beta$-mercaptoethanol (non reducing condition). Brain and spinal cord homogenates were separated on SDS 4-20\% gradient polyacrylamide gels (Biorad, CA, USA) and then electrotransferred onto PVDF membranes (Biorad, CA, USA). Non-specific binding sites were blocked with $5 \%$ nonfat dry milk in Tris-buffered saline for $1 \mathrm{~h}$ at room temperature and then were incubated overnight at $4^{\circ} \mathrm{C}$ with a 1/1000 dilution of antibodies directed against either total tau (DA9) [29], pathological tau conformers (MC1), Phospho-Akt (Ser473) (D9E) XP and Phospho-GSK-3 $\beta$ (Ser9) (D85E12) XP antibodies (Cell Signaling technology, MA, USA). The following day, the membranes were washed 5 times with distilled water for 30 minutes and then incubated for $1 \mathrm{~h}$ at room temperature with a horseradish peroxidaselinked secondary anti-mouse or anti-rabbit antibody (1:1000 dilution) ( Cell Signaling technology, MA, USA). The immunoreactive bands signal intensity were visualized and quantified by chemiluminescence imaging with the ChemiDocTM XRS (Bio-Rad, CA, USA). To adjust for protein loading variations from lane to lane, membranes were also immunoprobed (after stripping) with a 1/1000 dilution of a GAPDHHRP conjugated antibody (Santacruz, CA, USA) or actin antibody (Chemicon, CA, USA) which were used as a reference antibody to quantify the amount of proteins electrotransferred. Protein sizes were estimated using proteins markers (Precision Plus Protein Standard, Biorad, CA, USA) and the ChemiDocTM XRS (Bio-Rad, CA, USA).

\section{Statistical analyzes}

Results are expressed as the mean \pm SEM. Statistical analyzes were performed using SPSS V12.0.1 for Windows. Data were examined for assumption of normality using the Shapiro-Wilk statistic and for homogeneity of variance using the Levene's test. Statistical significance was determined by Student's t-test, univariate or repeated measures analysis of variance (ANOVA) where appropriate followed by post-hoc comparisons with Bonferroni corrections. For data not satisfying assumptions of normality and homogeneity of variance, a nonparametric Mann-Whitney test was used. P-values $<0.05$ were considered significant.

\section{Results}

\section{Effects of Anatabine on the behavior and motor impairments of Tg Tau P301S mice}

Tg Tau P301S received anatabine in their drinking water at a dosage of $20 \mathrm{mg} / \mathrm{Kg}$ of body weight $/ 24$ hours or regular drinking water (placebo) for a period of 40 days. This dosage was selected based on our previous studies showing that at this dose anatabine displays an anti-inflammatory activity in the CNS [21-23]. During the time course of the treatment (40 days), 25\% of Tg Tau P301S placebo mice became paralyzed ( 3 out of 12) whereas none ( 0 out of 11 ) in the anatabine treatment group developed paralysis during the study duration (Figure 1). All mice that displayed hind-limb paralysis were excluded from the behavioral studies. After 39 days of treatment with anatabine, nonparalyzed Tg Tau P301S were evaluated for clasping behavior in the hind-limb extension test and for motor coordination using the rotarod performance test. An extension reflex in the hind-limb is expected when a mouse is suspended by the tail, however partial or complete hind-limb retraction is commonly observed in mice with motor neuron disease [27]. Approximately $44 \%$ (4 out of 9 non-paralyzed mice) of the placebo group (33 week-old mice) elicited an abnormal hind-limb extension reflex when suspended by the tail compared to $18 \%$ (2 out of 11) of Tg Tau P301S mice treated with anatabine (Figure 1). In addition, Tg Tau P301S mice treated with anatabine showed an increased latency to fall ( $T$-test, $\mathrm{P}<0.05$ ) from an accelerating rotarod apparatus compared to $\mathrm{Tg}$ Tau $\mathrm{P} 301 \mathrm{~S}$ mice receiving regular drinking water (Figure 2) showing improved motor coordination. After 21 days of treatment with anatabine, 31 week-old Tg Tau P301S were tested in the elevated plus maze to assess the impact of anatabine on 
Citation: Paris D, Beaulieu-Abdelahad D, Ait-Ghezala G, Mathura V, Verma M, et al, (2014) Anatabine Attenuates Tau Phosphorylation and Oligomerization in P301S Tau Transgenic Mice. Brain Disord Ther 3:126. doi:10.4172/2168-975X.1000126

Page 4 of 11
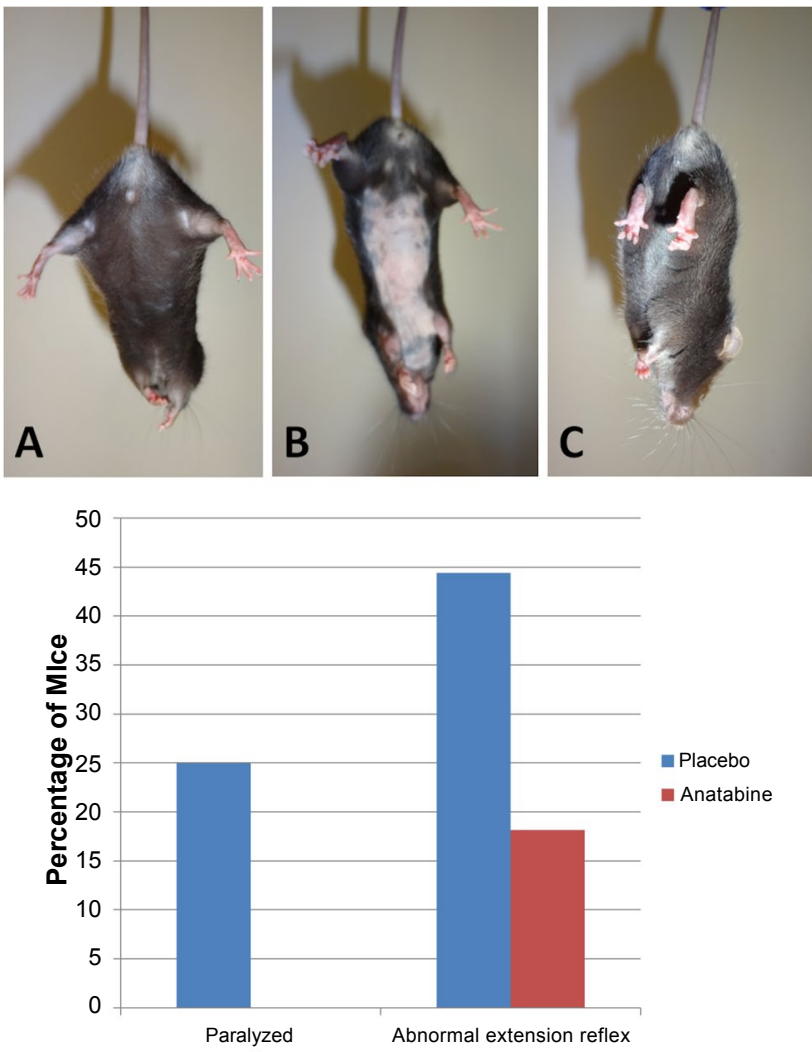

Figure 1: Impact of anatabine on the incidence of paralysis and abnormal hind-limb extension reflex in Tg Tau P301S. Representative Tg Tau P301S mice pictures showing a mouse with a normal hind-limb reflex (a) when suspended by the tail, an animal with a partial hind-limb extension reflex (b) and one mouse with a complete absence of extension reflex in both hind-limb (c). The histogram represents the proportion of Tg Tau P301S with complete posterior hind-limb paralysis and the proportion of non-paralyzed mice showing an abnormal hind-limb extension reflex in the placebo and anatabine treatment groups after 39 days of treatment.

anxiety related behavior. Tg Tau P301S mice treated with anatabine spent significantly more time (T-test, $\mathrm{P}<0.05)$ in the open arms of the elevated plus maze than placebo Tg Tau P301S mice (Figure 3). Spatial learning abilities which depend on hippocampal functions were assessed by the radial arm water maze (RAWM) [34] after 30 days of treatment with anatabine. Compared to age matched wild-type mice, 32 week-old Tg Tau P301S mice did not show spatial learning impairment in the RAWM (data not shown). A trend for a reduction in the number of errors elicited by anatabine treated Tg Tau P301S mice to locate the hidden platform in the RAWM was observed (data not shown).

\section{Effect of anatabine treatment on tau phosphorylation and pathological tau conformers}

The impact of the anatabine treatment on tau expression level was evaluated by western-blots in brain and spinal cord homogenates under denaturing and reducing conditions using the antibody DA9 which recognizes total tau [29]. No effect of the anatabine treatment was observed on tau expression (T-test, $\mathrm{P}>0.05$ ) in Tg Tau P301S mice (Figure 4). To assess the effect of anatabine on tau phosphorylation in the brain and spinal cord of Tg P301S mice, we used 3 different antibodies recognizing pathological $\mathrm{AD}$ relevant phosphorylated epitopes (PHF-1, RZ3 and CP13). The MC1 antibody was used to detect pathological tau conformers [29-31], whereas the antibody
TOC1 [33] was used to quantify tau oligomers. We employed a dotblot approach to quantify tau phosphorylation and pathological tau conformers using non-reducing and non-denaturing conditions with detergent soluble and insoluble fractions of brain and spinal cord homogenates. We validated our dot-blot approach for the detection of pathological tau hyperphosphorylation and pathological tau conformers/oligomers by using human brain homogenates from $\mathrm{AD}$ and non-demented individuals. Our dot-blot approach unambiguously allowed the identification of non-demented individuals from $\mathrm{AD}$ patients showing that it is a suitable method for the quantification of $\mathrm{AD}$ hyperphosphorylated tau and $\mathrm{AD}$ pathological tau conformers/ oligomers (Figure 5). In particular, T-test reveals statistically significant differences $(\mathrm{P}<0.001)$ for all the tau phosphorylated epitopes tested between $\mathrm{AD}$ and non-demented patients and a significant elevation in pathological tau conformer and tau oligomer levels $(\mathrm{P}<0.001)$ in brain homogenates from $\mathrm{AD}$ compared to non-demented patients.

In both the detergent soluble and insoluble fractions of the brain

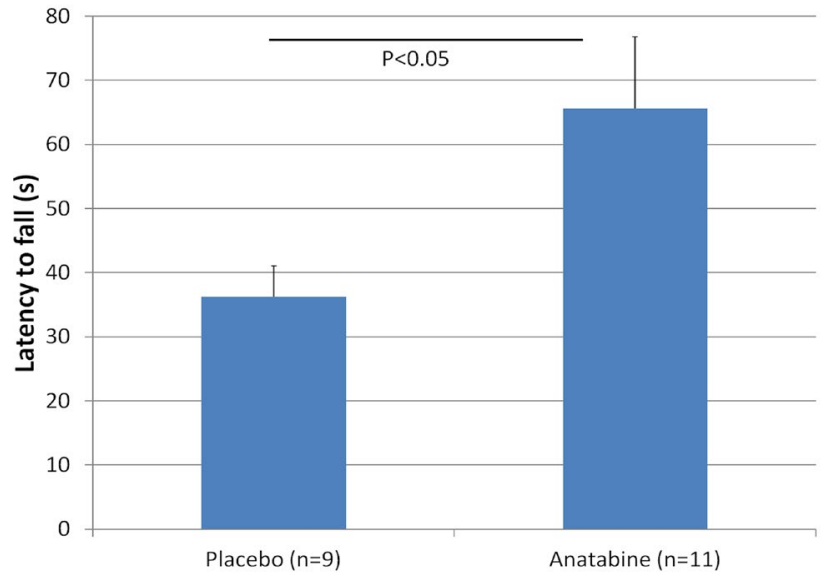

Figure 2: Chronic Treatment With Anatabine Improves Motor Performance In Tg Tau P301s Mice. The Histogram Depicts The Effect Of Anatabine On The Average Latency To Fall From An Accelerating Rotarod Apparatus After A Treatment Duration Of 39 Days.

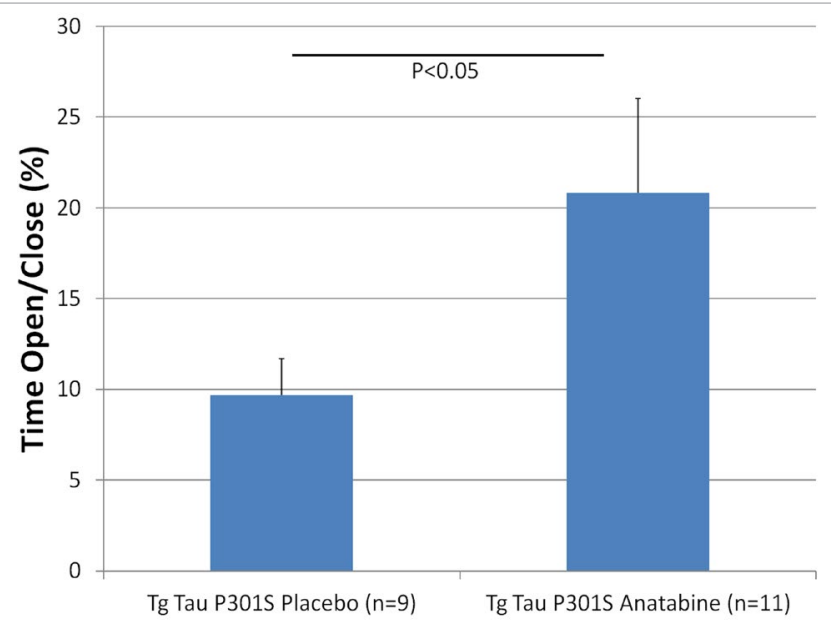

Figure 3: Effect of anatabine administration on anxiety assessed in the elevated plus maze. The histogram represents the average amount of time spent by $\mathrm{Tg}$ Tau P301S in the open arms of the elevated plus maze after a treatment duration of 21 days with anatabine. 
Citation: Paris D, Beaulieu-Abdelahad D, Ait-Ghezala G, Mathura V, Verma M, et al, (2014) Anatabine Attenuates Tau Phosphorylation and Oligomerization in P301S Tau Transgenic Mice. Brain Disord Ther 3:126. doi:10.4172/2168-975X.1000126

Page 5 of 11
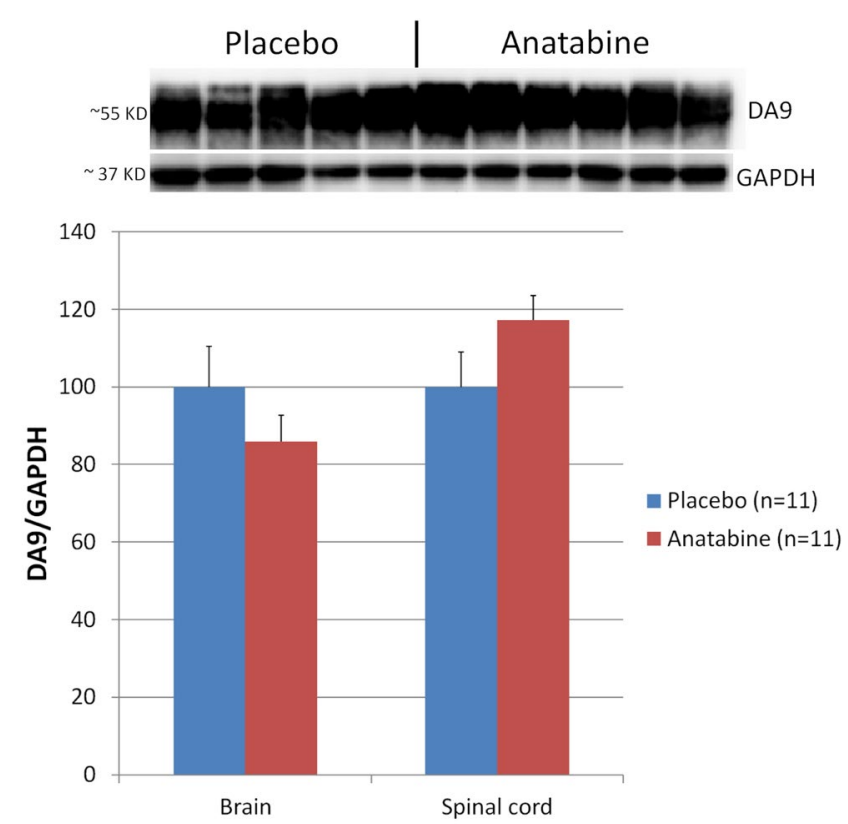

Figure 4: Impact of anatabine administration on total tau expression in the brain and spinal cord of $\mathrm{Tg} \mathrm{Tau}$ P301S. A representative western-blot depicting the amount of total tau (detected with the antibody DA9) in brain homogenates of placebo and anatabine treated $\mathrm{Tg} \mathrm{Tau}$ P301S mice is shown. GAPDH (Glyceraldehyde 3-phosphate dehydrogenase) was used as a reference protein to correct for possible unequal loading and electrotransfer from lane to lane. The histogram represents the average amount of total tau (DA9/GAPDH chemiluminescent signals) quantified in the brain and spinal cord homogenates of placebo and anatabine treated $\mathrm{Tg}$ P301S mice.

and spinal cord homogenates of Tg Tau P301S mice, we found that tau phosphorylation was significantly reduced $(\mathrm{T}$-tests, $\mathrm{P}<0.05)$ by the anatabine treatment for all the $\mathrm{AD}$ phosphorylated epitopes tested (Figure 6). Moreover, a significant reduction in MC1-immunoreactivity $(\mathrm{P}<0.05)$ was observed in the detergent soluble and insoluble fractions of brain and spinal cord homogenates from Tg Tau P301S mice treated with anatabine showing that anatabine prevents the formation of pathological tau conformers (Figure 7). Additionally, a significant reduction $(\mathrm{P}<0.05)$ in tau oligomers levels (TOC1 immunopositive) was detected both in the brain and the spinal cord of Tg Tau P301S treated with anatabine using dot-blots (Figure 8).

We also subjected the detergent soluble fractions of brain and spinal cord homogenates to western-blotting to detect tau oligomers using non-reducing conditions as tau oligomer formation involves disulfide cross-linking between tau molecules [30,35]. MC1 immunopositive tau oligomers were identified in brain and spinal cord homogenates from Tg Tau P301S mice by western-blotting whereas tau monomers ( $50-55 \mathrm{KD})$ were only weakly immunoreactive to the conformational antibody MC1 (Figure 9) under non-reducing condition. The most abundant tau oligomeric species immunopositive for MC1 detected in brain homogenates of Tg Tau P301S mice appear to migrate at an approximate size of 290 and $420 \mathrm{KDa}$. When the western-blots were overexposed additional MC1 immunopositive materials of an approximate size of 590, 160 and $100 \mathrm{KD}$ were also revealed (data not shown). We observed that anatabine significantly prevented the formation of MC1-positive tau oligomers (Figure 9) by western-blots in both the brain and the spinal cord of Tg Tau P301S mice (T-tests, $\mathrm{P}<0.05)$ further confirming the data obtained with the dot-blots. We evaluated the amount of Ibal immunoreactivity by dot-blotting in brain and spinal cord homogenates as a marker of microgliosis [3638]. A significant reduction in Ibal immunoreactivity was observed both in the brain $(\mathrm{T}$-test, $\mathrm{P}<0.01)$ and spinal cord (T-test, $\mathrm{P}<0.01$ ) homogenates of Tg Tau P301S mice treated with anatabine. Figure 10.

\section{Impact Of Anatabine On Brain Akt And Gsk3 $\beta$ Phosphorylation}

Glycogen synthase $3 \beta$ (GSK3 $\beta$ ) is one of the main serine-threonine kinase responsible for tau phosphorylation and has been shown to affect tau phosphorylation at multiple $\mathrm{AD}$ relevant epitopes including Ser396, Ser404, Thr231 and Ser202 [39-40]. GSK3 $\beta$ is inactivated
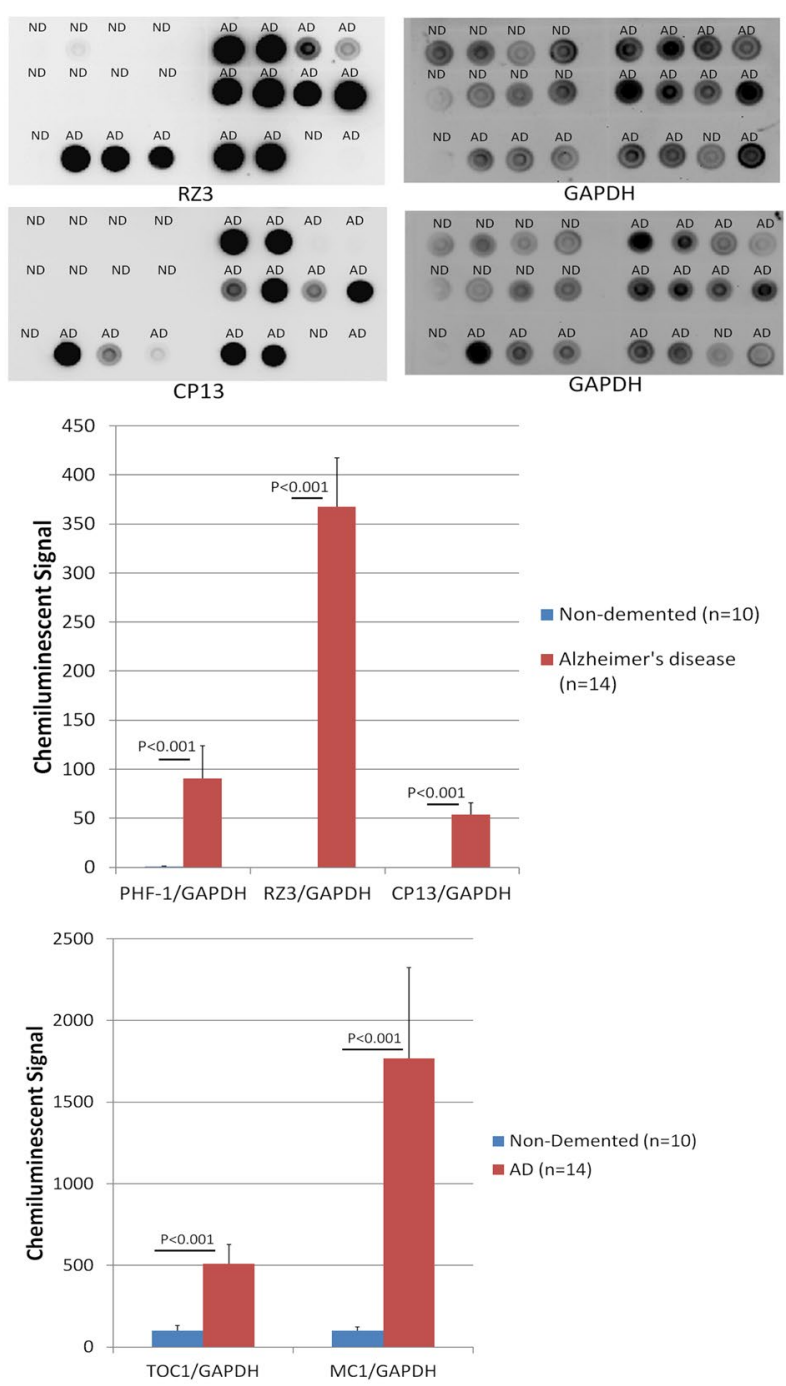

Figure 5: Quantification of tau hyperphosphorylation, pathological tau conformers and oligomers in brain homogenates from non-demented and $A D$ patients by dot-blots. Representative dot-blots revealing the amount of CP13 (Ser202) and RZ3 (Thr231) tau phosphorylation in brain homogenates from non-demented (ND) and AD patients (AD). Protein content in each sample was normalized using GAPDH as a reference protein. The histogram represent the average CP13/GAPDH, PHF-1 (Ser396/Ser404)/GAPDH, RZ3/GAPDH chemiluminescent signals quantified in brain homogenates from $A D$ and nondemented patients. Pathological tau conformer and tau oligomer levels were quantified with the antibodies MC1 and TOC1 respectively. The histogram represents the average MC1/GAPDH and TOC1/GAPDH chemiluminescent signals quantified in brain homogenates from $A D$ and non-demented patients. 
Citation: Paris D, Beaulieu-Abdelahad D, Ait-Ghezala G, Mathura V, Verma M, et al, (2014) Anatabine Attenuates Tau Phosphorylation and Oligomerization in P301S Tau Transgenic Mice. Brain Disord Ther 3:126. doi:10.4172/2168-975X.1000126

Brain Soluble Fraction

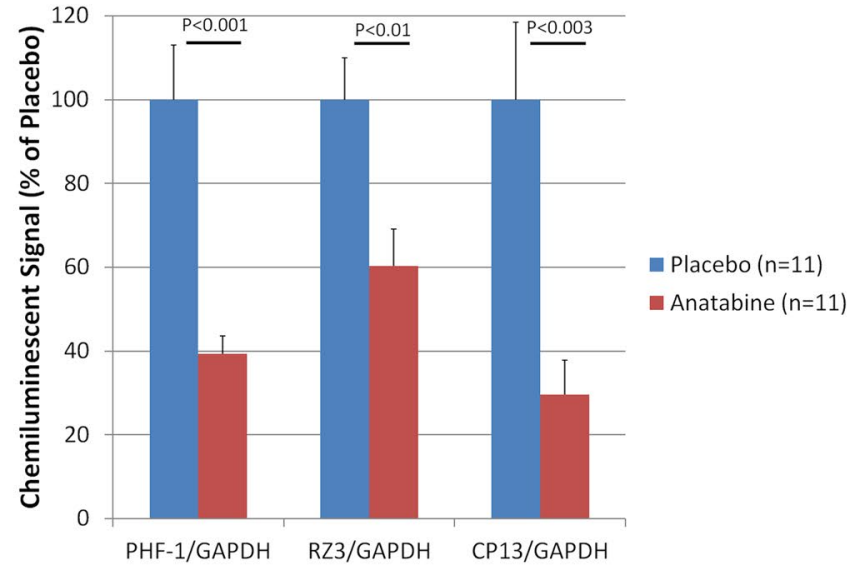

Spinal Cord Soluble Fraction

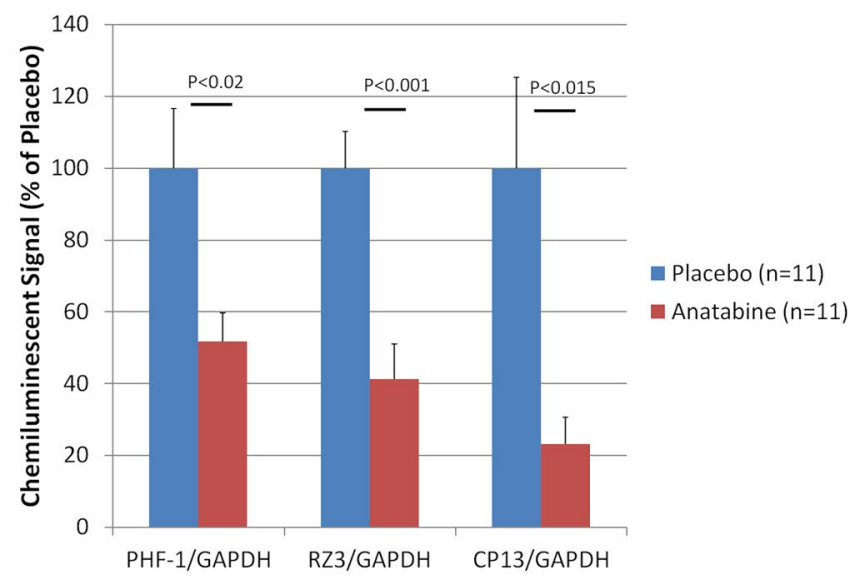

Brain Insoluble Fraction

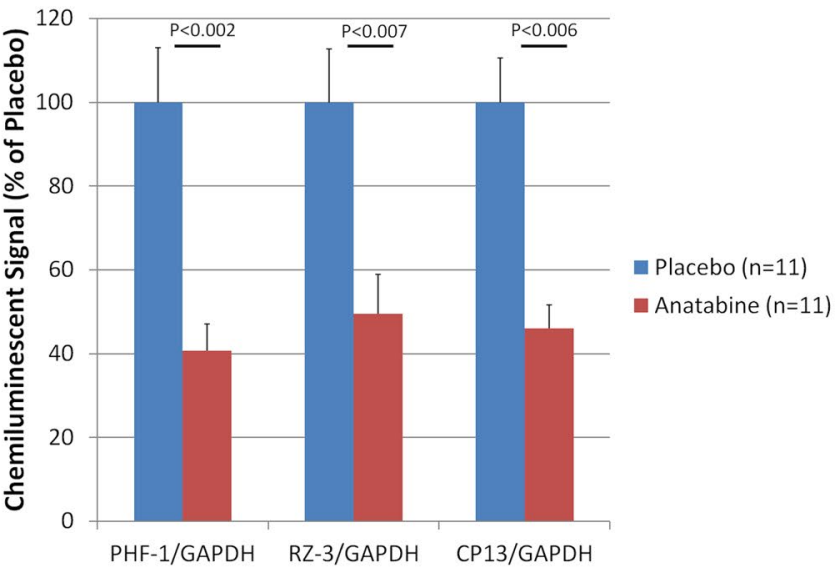

Spinal Cord Insoluble Fraction

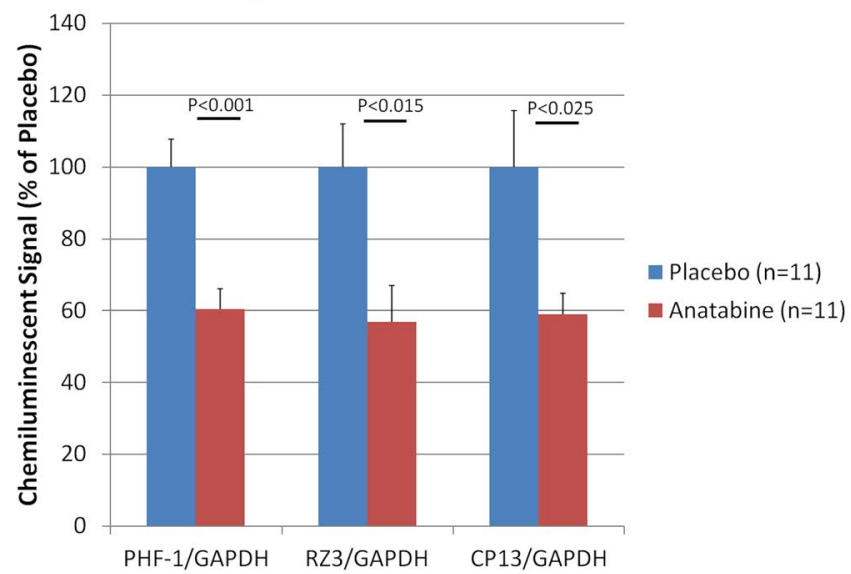

Figure 6: Effect of anatabine administration on the level of tau hyperphosphorylation in detergent soluble and insoluble fractions of the brain and spinal cord of Tg Tau P301S mice. The histograms represent the average values of PHF-1/GAPDH, RZ3/GAPDH and CP13/GAPDH chemiluminescent signals quantified by dot-blots in brain and spinal cord detergent soluble and detergent insoluble fractions.

upon phosphorylation of Ser9 by protein kinase B (AKT) [41] whereas AKT phosphorylation at Ser473 results in AKT activation [42]. We therefore evaluated the possible impact of the anatabine treatment on GSK3ßSer9 and AKT Ser473 phosphorylation. We found a significant increase in brain AKT Ser473 phosphorylation (T-test, $\mathrm{P}<0.001)$ and GSK $3 \beta$ Ser9 phosphorylation (T-test, $\mathrm{P}<0.001)$ in Tg Tau P301S mice treated with anatabine. Figure 11.

\section{Discussion}

We previously reported that anatabine lowers $A \beta$ production both in vitro and in vivo and readily crosses the blood-brain barrier, suggesting it could represent a potential compound for the treatment of $\mathrm{AD}$ [22]. While searching for the mechanism of action of anatabine, we delineated the signaling pathways impacted by this compound. In particular, we identified that anatabine prevents the activation of NFkB and STAT3 signaling pathways which are known to regulate inflammatory processes as well as the expression of BACE-1, the rate limiting enzyme responsible for the production of $A \beta$ peptides [2123]. Late stage $\mathrm{AD}$ clinical trial using compounds that directly target $A \beta$ peptides have been unsuccessful suggesting that once $A \beta$ deposition reaches a certain level targeting it may not be helpful. As AD is defined by the accumulation of $A \beta$ deposits and by the intraneuronal inclusion of hyperphosphorylated tau proteins, therapies targeting both $A \beta$ and tau pathologies are expected to be superior to therapeutic interventions solely targeting $A \beta$. Since anatabine prevents the phosphorylation of STAT3 and NFkB [21-23], we hypothesized that anatabine likely reduces the activity of kinases upstream of these transcription factors which may also be responsible for tau phosphorylation. We therefore investigated here the impact of anatabine on tau phosphorylation in a pure model of tauopathy (Tg Tau P301S mice) to determine whether this compound is capable of reducing both $A \beta$ and tau pathologies in preclinical models.

The anatabine treatment was initiated in 28 week-old ( $\sim 6$ and 1/2 months) Tg Tau P301S and lasted for 40 days. At that age, Tg Tau P301S mice (PS19 line) display hind-limb retraction when lifted by the tail which is indicative of disease progression in various mouse model of neurodegeneration [20,27]. This progresses to limb weakness, brain atrophy and eventually paralysis at 7 months of age in Tg Tau P301S mice [20]. Anatabine which is bioavailable orally was administered in the drinking water of the animals for a period of 40 

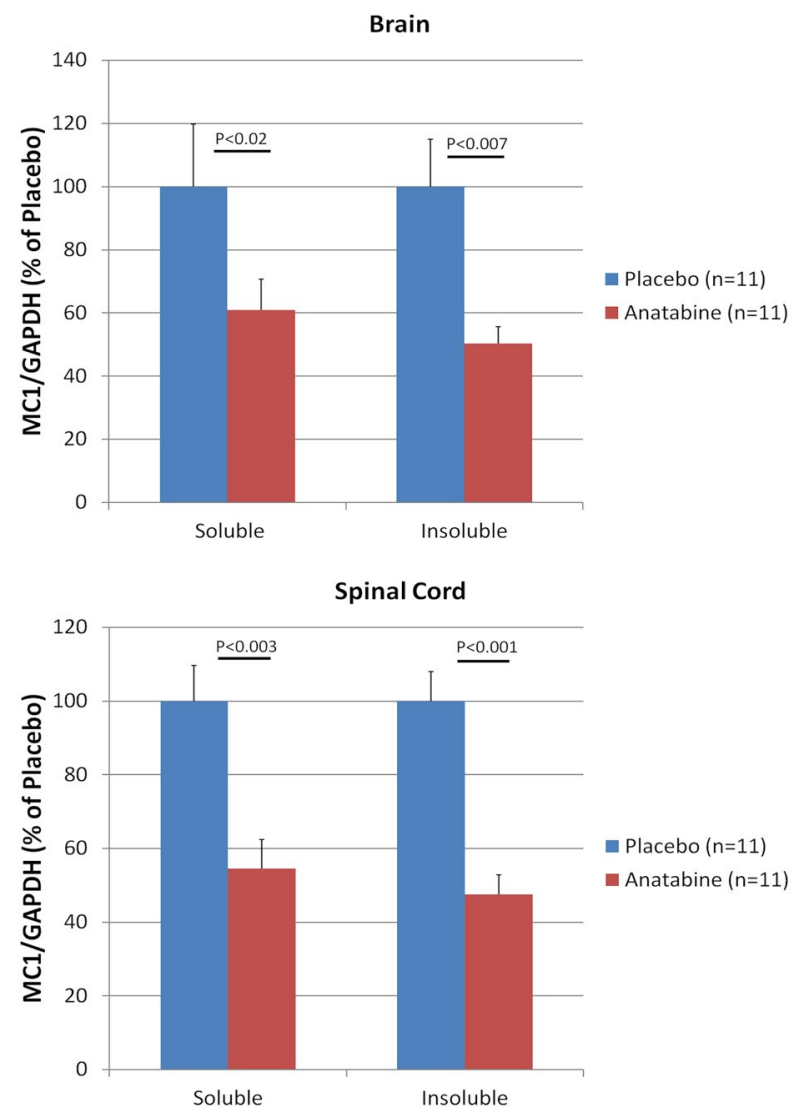

Figure 7: Impact of anatabine administration on the level of pathological tau conformers in the brain and spinal cord of Tg Tau P301S mice. The histograms represent the average pathological MC1/GAPDH chemiluminescent signal quantified in detergent soluble and insoluble fractions of brain and spinal cord homogenates.
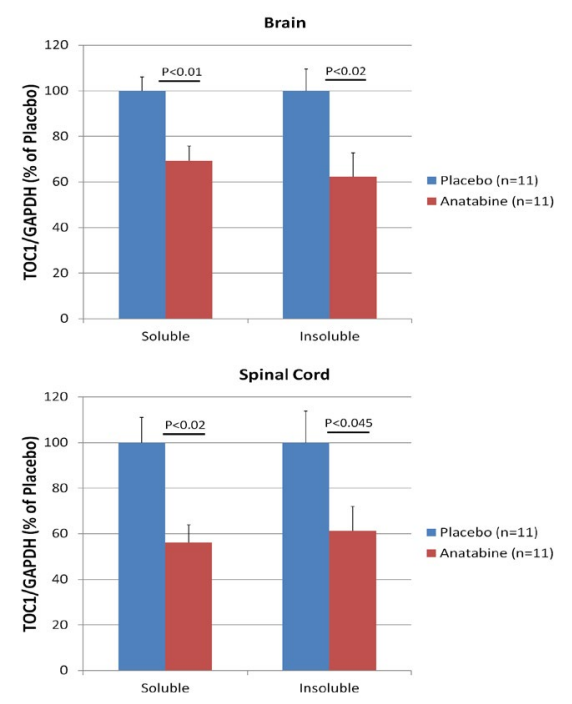

Figure 8: Effect of anatabine administration on the level of tau oligomers in the brain and spinal cord of $\mathrm{Tg} \mathrm{Tau}$ P301S mice. The histogram represents the average TOC1/GAPDH chemiluminescent signal quantified in detergent soluble and insoluble fractions of brain and spinal cord homogenates . days. During the treatment period, the behavior of Tg Tau P301S mice was evaluated using a battery of behavioral tests. Our data show that anatabine improves locomotor coordination in Tg Tau P301S mice since the latency to fall from an accelerating rotarod apparatus was significantly increased (by approximately 2 fold) in anatabine treated mice. In addition, abnormal hind-limb extension reflex and hindlimb paralysis were reduced by anatabine in Tg Tau P301S suggesting that anatabine could prevent disease progression in Tg Tau P301S mice although a larger cohort of mice will be required to confirm this observation. Examination of multiple pertinent $\mathrm{AD}$ phosphorylated tau epitopes show that anatabine reduces tau phosphorylation both in detergent soluble and insoluble fractions of the brain and spinal cord homogenates of Tg Tau P301S mice. We found in particular that tau phosphorylation at Ser396/Ser404 (PHF-1), Thr231 (RZ3) and Ser202 (CP13) was significantly reduced in the brain and spinal cord of Tg Tau P301S treated with anatabine. Our data are consistent with previous data showing that inhibition of tau hyperphosphorylation can prevent motor impairments and delay disease progression in transgenic mouse model of tauopathy [43] and suggest that the reduction in tau phosphorylation induced by anatabine could be responsible for the prevention of locomotor deficits in Tg Tau P301S mice. We did not find that spatial learning was impaired in Tg Tau P301S compared to wild-type mice using the Morris water maze or the radial arm water maze (RAWM) (data not shown) in agreement with previous reports showing an absence of cognitive impairment or minimal alteration of cognition despite extensive tau pathology and presynaptic pathology in hippocampal neurons in various mouse model of tauopathy $[44,45]$. A trend for a reduction of the number of errors to locate the hidden platform in the RAWM was observed in anatabine treated $\mathrm{Tg}$ Tau P301S mice suggesting that anatabine may facilitate spatial learning in Tg Tau P301S mice (data not shown). In addition, we found that anatabine prolongs the time spent by Tg Tau P301S in the open arms of the elevated plus maze suggesting that anatabine reduces anxietylike behavior.

Several studies have shown that soluble oligomers of tau (rather than high molecular mass aggregates of tau present in neurofibrillary tangle) are the main tau pathological species responsible for neurodegeneration $[32,43,46,47]$. In particular, passive immunization using the conformational antibody $\mathrm{MC} 1$ appears superior at reducing tau pathology and hind-limb weakness in a mouse model of tauopathy than a high affinity pan-tau antibody $[48,49]$ highlighting the importance of MC1 pathological tau conformers in the development of neurodegenerative tauopathy. Interestingly, $\mathrm{MC1}$ recognizes pathological conformations of tau in the brain that occur at an early stage of $\mathrm{AD}$ preceding tau aggregation in neurofibrillary tangles $[11,31,50,51]$. In addition, passive immunization with a selective tau oligomer antibody has been shown to reverse taupathy phenotypes without affecting neurofibrillary tangles further emphasizing the critical role of tau oligomers in the development of tauopathy [52]. Using a selective oligomeric tau antibody (TOC1) which preferentially recognizes tau oligomers over monomers or aggregated polymers [33] , we observed a reduction in TOC1 imunoreactivity in the brain and spinal cord of Tg Tau P301S mice treated with anatabine. We also tested the impact of anatabine on the levels of MC1 pathological tau conformers in the brain and spinal cord of Tg Tau P301S mice by dot blotting. Our data show that anatabine reduces the amount of $\mathrm{MC1}$ pathological tau conformers in both the brain and spinal cord of Tg Tau P301S mice. Western-blot analysis of brain and spinal cord homogenates from Tg Tau P301S under non-reducing condition reveals the presence of $\mathrm{MC1}$ immunopositive tau oligomers. The 
Citation: Paris D, Beaulieu-Abdelahad D, Ait-Ghezala G, Mathura V, Verma M, et al, (2014) Anatabine Attenuates Tau Phosphorylation and Oligomerization in P301S Tau Transgenic Mice. Brain Disord Ther 3:126. doi:10.4172/2168-975X.1000126
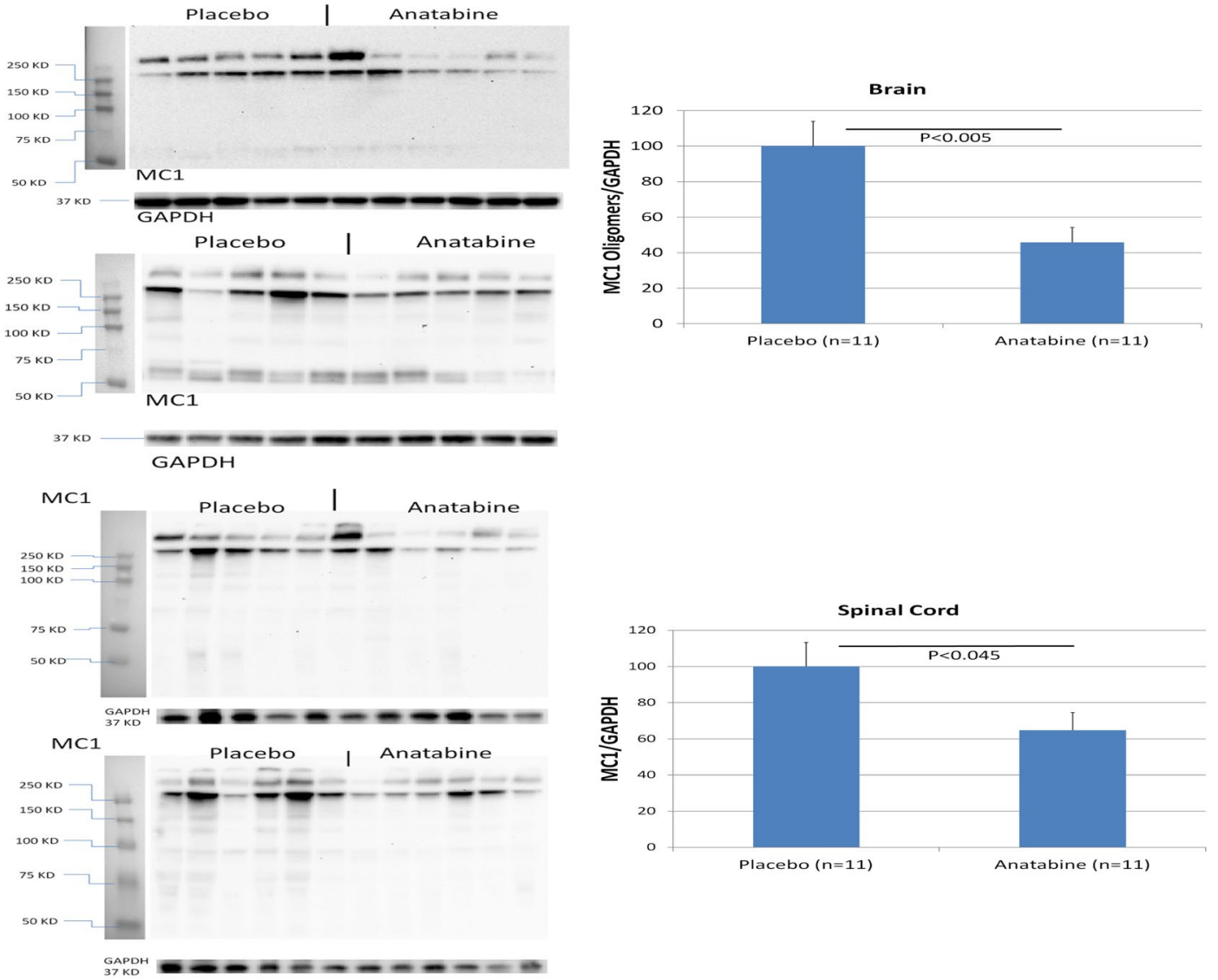

Figure 9: Quantification of MC1 immunopositive tau oligomers by western-blots in the brain and spinal cord of Tg Tau P301S. Representative western-blots revealing MC1 immunopositive tau oligomers in the brain and spinal cord (detergent soluble fraction, non-reducing conditions were used) of Tg Tau P301S of the placebo and anatabine treatment group. The histogram represents the quantification of MC1 tau oligomers in the brain and spinal cord of Tg Tau P301S mice.
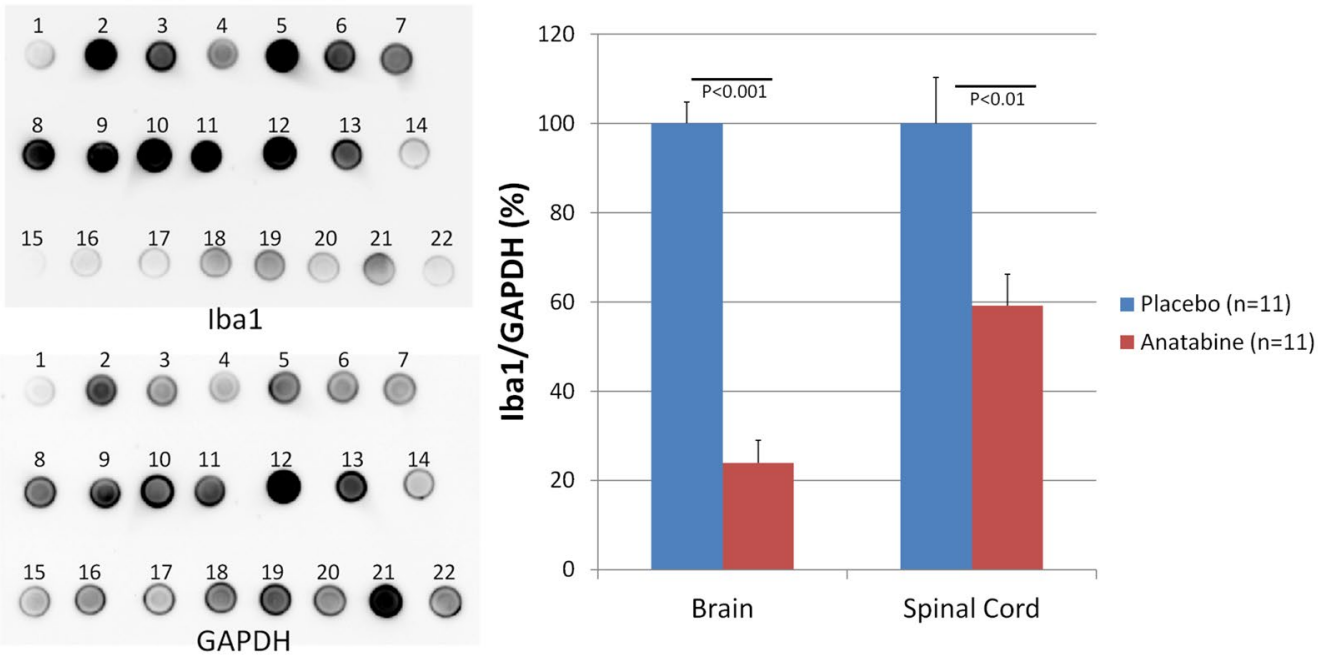

Figure 10: Effect of the anatabine treatment on brain and spinal cord lba1 levels. A) A representative dot blot showing the level of Iba1 and GAPDH (as a reference protein) in brain homogenates from placebo Tg Tau P301S mice (1 to 11) and from Tg Tau P301S mice treated with anatabine (12 to 22) is shown. B) The histogram represents the quantification of Iba1/GAPDH chemiluminescent signal observed in the brain and spinal cord homogenates of Tg Tau P301S receiving the placebo and anatabine. 
Citation: Paris D, Beaulieu-Abdelahad D, Ait-Ghezala G, Mathura V, Verma M, et al, (2014) Anatabine Attenuates Tau Phosphorylation and Oligomerization in P301S Tau Transgenic Mice. Brain Disord Ther 3:126. doi:10.4172/2168-975X.1000126
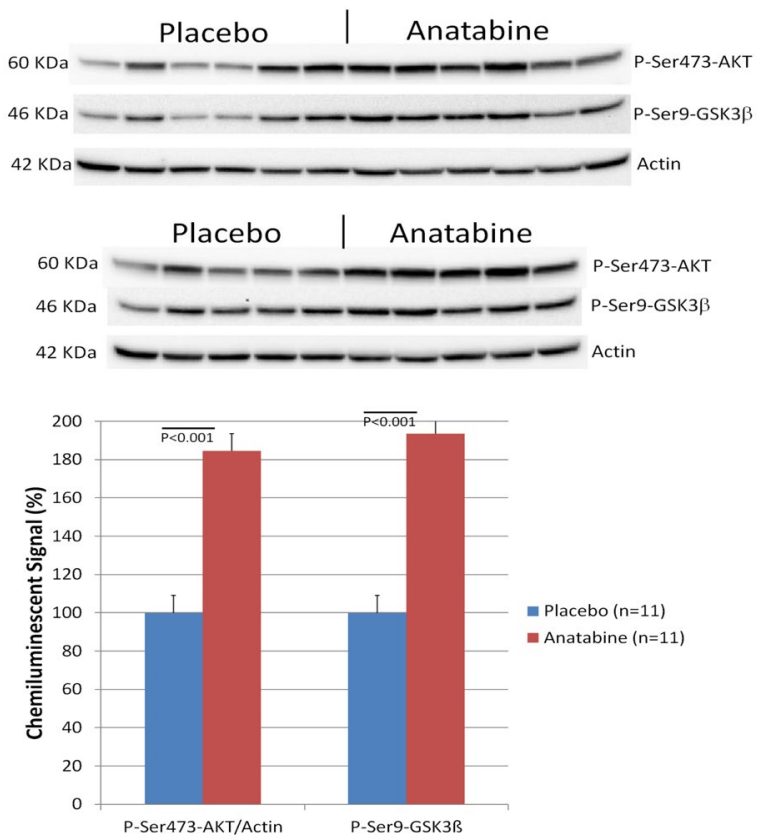

Figure 11: Effect of anatabine on the activation of AKT and GSK3 $\beta$ in the brain of $\mathrm{Tg} \mathrm{Tau}$ P301S mice. A representative western-blot of brain homogenates from placebo and anatabine treated $\mathrm{Tg}$ Tau P301S mice showing phosphorylated AKT at Ser473, phosphorylated GSK3 $\beta$ at Ser9 and actin as a reference protein is shown. The histogram represents the average values of phosphorylated AKT/Actin and phosphorylated GSK3ß/Actin for the brains of $\mathrm{Tg} \mathrm{Tau}$ P301S mice of the placebo and anatabine treatment groups.

levels of these MC1 tau oligomers detected by Western-blots appear significantly decreased in the brain and spinal cord of anatabine treated Tg Tau P301S mice further confirming the data obtained with the dot blot approach showing a reduction in tau oligomers in anatabine treated mice. While anatabine reduces tau phosphorylation and tau oligomers in detergent soluble and insoluble fractions of brain homogenates, further work will be required to determine whether anatabine can also prevent the formation of neurofibrillary tangle although it is apparent that tau oligomers rather than neurofibrillary tangles plays a critical role in disease progression [52].

Microglial activation is one of the earliest brain pathologies in $\mathrm{Tg}$ Tau P301S and has been shown to correlate with hipocampal atrophy in this tauopathy model [20]. Interestingly, inhibition of microglial activation using an immunosuppressant prevented hipocampal atrophy, neuronal loss as well as tau hyperphosphorylation in Tg Tau P301S mice [20] suggesting a mechanistic link between microglial activation and the progression of tau pathologies. We therefore evaluated Iba1 level in brain and spinal cord homogenates of Tg Tau P301S mice as Ibal expression is increased in activated microglia [36]. We observed that anatabine significantly suppressed Iba1 expression in the brain and spinal cord of $\mathrm{Tg}$ Tau P301S suggesting that anatabine is opposing microglial activation in $\mathrm{Tg}$ Tau P301S mice. The reduction in microgliosis induced by anatabine in Tg P301S mice may be consequential to the reduction of tau phosphorylation and oligomerization. We have shown however, that in a mouse model of multiple sclerosis, anatabine can mitigate microgliosis in the brain and the spinal cord [23] which could suggest that anatabine can directly oppose microgliosis in Tg Tau P301S mice. Microgliosis occurs prior to any significant tau pathology in Tg Tau P301S mice and also contributes to tau phosphorylation and oligomerization [20]. The anti-inflammatory activity of anatabine may therefore also play an important role in the mitigation of tau phosphorylation and oligomerization observed after anatabine treatment in Tg Tau P301S mice.

Anatabine displays a chemical structure closely related to nicotine and is therefore anticipated to act as an agonist of nicotinic acethylcholine receptors (nAChR). Our previous data showing that anatabine inhibits both STAT3 and NFkB phosphorylation [21-23] support this contention as nAChR agonists typically elicit an anti-inflammatory activity by blunting both STAT3 and NFkB activation [53-55]. Recent data have shown that anatabine is a preferential $\alpha 7 \mathrm{nAChR}$ agonist and a weaker or partial $\alpha 4 \beta 2 \mathrm{nAChR}$ agonist [56]. Interestingly, selective $\alpha 7 \mathrm{nAChR}$ agonists have been shown to prevent tau phosphorylation both in vitro and in vivo by stimulating protein kinase B (AKT) resulting in the inactivation of glycogen synthase kinase $3 \beta$, one of the main kinase responsible for tau hyperphosphorylation [57-59]. We therefore investigated the possible impact of anatabine on AKT and GSK3 $\beta$ activation in the brain of Tg Tau P301S mice. We observed an activation of AKT (increased phosphorylation at Ser473) as well as an increased in GSK3 $\beta$ phosphorylation at Ser9 (indicative of GSK3 $\beta$

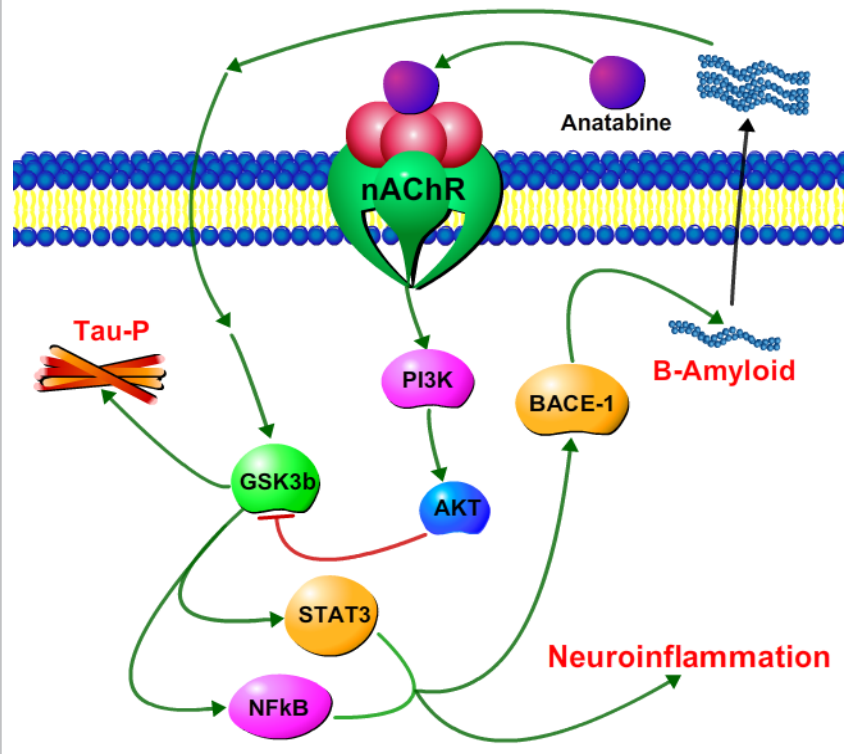

Figure 12: Proposed hypothesis for the mechanism of action of anatabine in $A D$. In $A D$, excessive accumulation of $A \beta$ peptides results in GSK3 $\beta$ activation and tau hyperphosphorylation, followed by tau oligomerization and neurodegeneration. Increased GSK3 $\beta$ activity in AD will also induce the activation of the transcription factors STAT3 and NFKB which have central roles in inflammatory reactions, regulating many proteins involved in neuroinflammation including cytokines. Additionally, STAT3 and NFKB activation will induce BACE1 expression (the rate limiting enzyme responsible for $A \beta$ production), possibly resulting in a self propagating pathway contributing to both $A \beta$ production/ accumulation, neuroinflammation and tau hyperphosphorylation. Upon binding of anatabine to nicotinic acetylcholine receptors ( $\mathrm{ACChR}$ ) and stimulation, the survival signal transduction pathway PI3K and AKT is activated, leading to an increased AKT dependent Ser9 phosphorylation of GSK3 $\beta$ which dominantly inhibits its activity, resulting in decreased tau phosphorylation at multiple serine and threonine residues. Following the inactivation of GSK3 $\beta$ by anatabine, a decreased activation of STAT3 and NFKB will occur, resulting in decreased neuroinflammation, reduced BACE- 1 expression and $A \beta$ production. It is therefore anticipated that anatabine has the potential to mitigate the three main pathologies associated with $A D$ : tau hyperphosphorylation, $A \beta$ accumulation and neuroinflammation. 
Citation: Paris D, Beaulieu-Abdelahad D, Ait-Ghezala G, Mathura V, Verma M, et al, (2014) Anatabine Attenuates Tau Phosphorylation and Oligomerization in P301S Tau Transgenic Mice. Brain Disord Ther 3:126. doi:10.4172/2168-975X.1000126

inactivation) in the brain of Tg Tau P301S mice treated with anatabine which could explain the reduction in tau phosphorylation observed in anatabine treated mice and provide support for a mechanism involving a stimulation of $\alpha 7 \mathrm{nAChR}$. A $50 \%$ increase in the phosphorylation of the inhibitory residue (Ser9) of GSK3 $\beta$ was also observed in the spinal cord of Tg Tau P301S mice treated with anatabine (data not shown). We have shown that anatabine opposes neuroinflammation by preventing the activation of NFkB and STAT3 [21-23]. It is likely that the inhibition of STAT3 and NFkB observed is consequent to the inactivation of GSK3 $\beta$ by anatabine, as both STAT3 and NFkB activation are highly dependent on GSK3 $\beta$ activity [60,61]. We have summarized on Figure 12 a possible mechanism of action for anatabine that highlights its potential therapeutical application in AD. Further mechanistic work will be required to precisely identify the mode of action of anatabine responsible for the prevention of tau phosphorylation and determine whether it is mainly mediated via a reduction of microgliosis/neuroinflammation, an inhibition of the kinases responsible for tau phosphorylation or a combination of both.

Our data provide in vivo evidence that a chronic oral treatment with anatabine reduces pathological tau hyperphosphorylation and the formation of pathological tau conformers/oligomers leading to a reduction of tau-related functional deficits in Tg Tau P301S mice. These data support further exploration of anatabine as a possible disease modifying agent for tau-associated neurodegenerative conditions and, in particular $\mathrm{AD}$, since we have shown that anatabine also displays $\mathrm{A} \beta$ lowering properties [22].

\section{Acknowledgements}

We are grateful to Dr. Peter Davies (Albert Einstein College of Medecine, Bronx, NY, USA) for the gift of the DA9, PHF-1, CP13, RZ3 and MC1 antibodies and to Dr. Lester Binder (Feinberg School of Medicine, Northwestern University, Chicago, IL, USA) for providing the TOC1 antibody. AER is the recipient of an NIA grant: RO1 AG-19795. We thank Mr. Thomas Guengant for his help during the behavioral testing.

\section{References}

1. Takata K, Kitamura $Y$ (2012) Molecular approaches to the treatment prophylaxis, and diagnosis of Alzheimer's disease: tangle formation, amyloid- $\hat{I}^{2}$, and microglia in Alzheimer's disease. J Pharmacol Sci 118: 331-337.

2. Perry E, Walker M, Grace J, Perry R (1999) Acetylcholine in mind: neurotransmitter correlate of consciousness? Trends Neurosci 22: 273-280.

3. Szot P (2012) Common factors among Alzheimer's disease, Parkinson's disease, and epilepsy: possible role of the noradrenergic nervous system. Epilepsia 53 Suppl 1: 61-66.

4. Femminella GD, Rengo G, Pagano G, de Lucia C, Komici K, et al. (2013) $\hat{I}^{2}$ adrenergic receptors and $G$ protein-coupled receptor kinase-2 in Alzheimer's disease: a new paradigm for prognosis and therapy? J Alzheimers Dis 34: 341 347.

5. Takahashi M, Uchikado H, Caprotti D, Weidenheim KM, Dickson DW, et al (2006) Identification of G-protein coupled receptor kinase 2 in paired helical filaments and neurofibrillary tangles. J Neuropathol Exp Neurol 65: 1157-1169.

6. Wan J, Fu AK, Ip FC, Ng HK, Hugon J, et al. (2010) Tyk2/STAT3 signaling mediates beta-amyloid-induced neuronal cell death: implications in Alzheimer's disease. J Neurosci 30: 6873-6881.

7. Camandola S, Mattson MP (2007) NF-kappa B as a therapeutic target in neurodegenerative diseases. Expert Opin Ther Targets 11: 123-132.

8. Xiong J, Wang C, Chen $\mathrm{H}, \mathrm{Hu}$ Y, Tian L, et al. (2013) $A \hat{I}^{2}$-induced microglial cell activation is inhibited by baicalin through the JAK2/STAT3 signaling pathway. Int $\mathrm{J}$ Neurosci

9. Capiralla H, Vingtdeux V, Zhao H, Sankowski R, Al-Abed Y, et al. (2012) Resveratrol mitigates lipopolysaccharide- and $A \hat{I}^{2}$-mediated microglial inflammation by inhibiting the TLR4/NF-Î'B/STAT signaling cascade. J Neurochem 120: 461-472.
10. Bertram L, Lill CM, Tanzi RE (2010) The genetics of Alzheimer disease: back to the future. Neuron 68: 270-281.

11. Haroutunian V, Davies P, Vianna C, Buxbaum JD, Purohit DP (2007) Tau protein abnormalities associated with the progression of alzheimer disease type dementia. Neurobiol Aging 28: 1-7.

12. Tremblay C, Pilote M, Phivilay A, Emond V, Bennett DA, et al. (2007) Biochemical characterization of Abeta and tau pathologies in mild cognitive impairment and Alzheimer's disease. J Alzheimers Dis 12: 377-390.

13. Vandrovcova J, Anaya F, Kay V, Lees A, Hardy J, et al. (2010) Disentangling the role of the tau gene locus in sporadic tauopathies. Curr Alzheimer Res 7: 726-734.

14. Myers AJ, Pittman AM, Zhao AS, Rohrer K, Kaleem M, et al. (2007) The MAPT H1c risk haplotype is associated with increased expression of tau and especially of 4 repeat containing transcripts. Neurobiol Dis 25: 561-570.

15. Reiman EM, Webster JA, Myers AJ, Hardy J, Dunckley T, et al. (2007) GAB2 alleles modify Alzheimer's risk in APOE epsilon4 carriers. Neuron 54: 713-720.

16. Martin L, Latypova X, Wilson CM, Magnaudeix A, Perrin ML et al. (2013) Tau protein kinases: involvement in Alzheimer's disease. Ageing Res Rev 12: 289309

17. Hernandez F, Lucas JJ, Avila J (2013) GSK3 and tau: two convergence points in Alzheimer's disease. J Alzheimers Dis 33 Suppl 1: S141-144.

18. Berger Z, Roder H, Hanna A, Carlson A, Rangachari V, et al. (2007) Accumulation of pathological tau species and memory loss in a conditional model of tauopathy. J Neurosci 27: 3650-3662.

19. Santacruz K, Lewis J, Spires T, Paulson J, Kotilinek L, et al. (2005) Tau suppression in a neurodegenerative mouse model improves memory function. Science 309: 476-481.

20. Yoshiyama Y, Higuchi M, Zhang B, Huang SM, Iwata N, et al. (2007) Synapse loss and microglial activation precede tangles in a P301S tauopathy mouse model. Neuron 53: 337-351.

21. Paris D, Beaulieu-Abdelahad D, Abdullah L, Bachmeier C, Ait-Ghezala G et al. (2013) Anti-inflammatory activity of anatabine via inhibition of STAT3 phosphorylation. Eur J Pharmacol 698: 145-153.

22. Paris D, Beaulieu-Abdelahad D, Bachmeier C, Reed J, Ait-Ghezala G, et al (2011) Anatabine lowers Alzheimer's $A \hat{l}^{2}$ production in vitro and in vivo. Eur J Pharmacol 670: 384-391.

23. Paris D, Beaulieu-Abdelahad D, Mullan M, Ait-Ghezala G, Mathura V, et al. (2013) Amelioration of experimental autoimmune encephalomyelitis by anatabine. PLoS One 8: e55392.

24. Caturegli $P$, De Remigis $A$, Ferlito $M$, Landek-Salgado MA, Iwama $S$, et al. (2012) Anatabine ameliorates experimental autoimmune thyroiditis. Endocrinology 153: 4580-4587.

25. Schmeltz LR, Blevins TC, Aronoff SL, Ozer K, Leffert JD, et al. (2013) Anatabine supplementation decreases thyroglobulin antibodies in patients with chronic lymphocytic autoimmune (Hashimoto's) thyroiditis: A randomized controlled clinical trial. J Clin Endocrinol Metab 99: E137-42.

26. Lanier RK, Gibson KD, Cohen AE, Varga M (2013) Effects of dietary supplementation with the solanaceae plant alkaloid anatabine on joint pain and stiffness: results from an internet-based survey study. Clin Med Insights Arthritis Musculoskelet Disord 6: 73-84.

27. Takahashi E, Niimi K, Itakura C (2009) Motor coordination impairment in aged heterozygous rolling Nagoya, Cav2.1 mutant mice. Brain Res 1279: 50-57.

28. File SE, Pellow S (1985) The effects of triazolobenzodiazepines in two animal tests of anxiety and in the holeboard. Br J Pharmacol 86: 729-735.

29. Acker CM, Forest SK, Zinkowski R, Davies P, d'Abramo C (2013) Sensitive quantitative assays for tau and phospho-tau in transgenic mouse models. Neurobiol Aging 34: 338-350.

30. Jeganathan S, Hascher A, Chinnathambi S, Biernat J, Mandelkow EM, et al (2008) Proline-directed pseudo-phosphorylation at AT8 and PHF1 epitopes induces a compaction of the paperclip folding of Tau and generates a pathological (MC-1) conformation. J Biol Chem 283: 32066-32076.

31. Weaver CL, Espinoza M, Kress Y, Davies P (2000) Conformational change as one of the earliest alterations of tau in Alzheimer's disease. Neurobiol Aging 21: $719-727$ 
Citation: Paris D, Beaulieu-Abdelahad D, Ait-Ghezala G, Mathura V, Verma M, et al, (2014) Anatabine Attenuates Tau Phosphorylation and Oligomerization in P301S Tau Transgenic Mice. Brain Disord Ther 3:126. doi:10.4172/2168-975X.1000126

Page 11 of 11

32. Patterson KR, Remmers C, Fu Y, Brooker S, Kanaan NM, et al. (2011) Characterization of prefibrillar Tau oligomers in vitro and in Alzheimer disease. J Biol Chem 286: 23063-23076.

33. Ward SM, Himmelstein DS, Lancia JK, Fu Y, Patterson KR, et al. (2013) TOC1: characterization of a selective oligomeric tau antibody. J Alzheimers Dis 37: 593-602.

34. Diamond DM, Park CR, Heman KL, Rose GM (1999) Exposing rats to a predator impairs spatial working memory in the radial arm water maze. Hippocampus 9 : $542-552$.

35. Sahara N, DeTure M, Ren Y, Ebrahim AS, Kang D, et al. (2013) Characteristics of TBS-extractable hyperphosphorylated tau species: aggregation intermediates in rTg4510 mouse brain. J Alzheimers Dis 33: 249-263.

36. Frank MG, Barrientos RM, Watkins LR, Maier SF (2010) Aging sensitizes rapidly isolated hippocampal microglia to LPS ex vivo. J Neuroimmunol 226: 181-184.

37. Sultan S, Gebara E, Toni N (2013) Doxycycline increases neurogenesis and reduces microglia in the adult hippocampus. Front Neurosci 7: 131.

38. Wirenfeldt M, Clare R, Tung S, Bottini A, Mathern GW, et al. (2009) Increased activation of Iba1+ microglia in pediatric epilepsy patients with Rasmussen's encephalitis compared with cortical dysplasia and tuberous sclerosis complex. Neurobiol Dis 34: 432-440.

39. Mandelkow EM, Drewes G, Biernat J, Gustke N, Van Lint J, et al. (1992) Glycogen synthase kinase-3 and the Alzheimer-like state of microtubuleassociated protein tau. FEBS Lett 314: 315-321.

40. Sperber BR, Leight S, Goedert M, Lee VM (1995) Glycogen synthase kinase-3 beta phosphorylates tau protein at multiple sites in intact cells. Neurosci Lett 197: 149-153.

41. Cross DA, Alessi DR, Cohen $P$, Andjelkovich M, Hemmings BA (1995) Inhibition of glycogen synthase kinase-3 by insulin mediated by protein kinase B. Nature 378: 785-789.

42. Alessi DR, Andjelkovic M, Caudwell B, Cron P, Morrice N, et al. (1996) Mechanism of activation of protein kinase $B$ by insulin and IGF-1. EMBO J 15: 6541-6551.

43. Le Corre S, Klafki HW, Plesnila N, Hübinger G, Obermeier A, et al. (2006) An inhibitor of tau hyperphosphorylation prevents severe motor impairments in tau transgenic mice. Proc Natl Acad Sci U S A 103: 9673-9678.

44. Harris JA, Koyama A, Maeda S, Ho K, Devidze N, et al. (2012) Human P301Lmutant tau expression in mouse entorhinal-hippocampal network causes tau aggregation and presynaptic pathology but no cognitive deficits. PLoS One 7: e45881.

45. Takeuchi H, Iba M, Inoue H, Higuchi M, Takao K, et al. (2011) P301S mutant human tau transgenic mice manifest early symptoms of human tauopathies with dementia and altered sensorimotor gating. PLoS One 6: e21050.

46. Lasagna-Reeves CA, Castillo-Carranza DL, Sengupta U, Guerrero-Munoz MJ, Kiritoshi T, et al. (2012) Alzheimer brain-derived tau oligomers propagate pathology from endogenous tau. Sci Rep 2: 700.

47. Davidowitz EJ, Chatterjee I, Moe JG (2008) Targeting tau oligomers for therapeutic development for Alzheimer's disease and tauopathies. Biotechnology 4: 47-64.
48. Asuni AA, Boutajangout A, Quartermain D, Sigurdsson EM (2007) Immunotherapy targeting pathological tau conformers in a tangle mouse mode reduces brain pathology with associated functional improvements. The Journal of Neuroscience 27: 9115-9129.

49. d'Abramo C, Acker CM, Jimenez HT, Davies P (2013) Tau passive immunotherapy in mutant $\mathrm{P} 301 \mathrm{~L}$ mice: antibody affinity versus specificity. PLoS One 8: e62402.

50. Mufson EJ, Ward S, Binder L (2014) Prefibrillar tau oligomers in mild cognitive impairment and Alzheimer's disease. Neurodegener Dis 13: 151-153.

51. Jicha GA, Lane E, Vincent I, Otvos L Jr, Hoffmann R, et al. (1997) A conformation- and phosphorylation-dependent antibody recognizing the paired helical filaments of Alzheimer's disease. J Neurochem 69: 2087-2095.

52. Castillo-Carranza DL, Sengupta U, Guerrero-Munoz, Lasagna-Reeves CA Gerson JE, et al. (2014) Passive immunization with tau oligomer monoclonal antibody reverses tauopathy phenotypes without affecting hyperphosphorylated neurofibrillary tangles. J Neurosci 34: 4260-4272.

53. Filippini P, Cesario A, Fini M, Locatelli F, Rutella S (2012) The Yin and Yang of non-neuronal $\mathbf{I} \pm 7$-nicotinic receptors in inflammation and autoimmunity. Curr Drug Targets 13: 644-655.

54. Hosur V, Loring RH (2011) $\hat{I} \pm 4 \hat{l}^{2} 2$ nicotinic receptors partially mediate antiinflammatory effects through Janus kinase 2-signal transducer and activator of transcription 3 but not calcium or cAMP signaling. Mol Pharmacol 79: 167-174.

55. Peña G, Cai B, Liu J, van der Zanden EP, Deitch EA, et al. (2010) Unphosphorylated STAT3 modulates alpha 7 nicotinic receptor signaling and cytokine production in sepsis. Eur J Immunol 40: 2580-2589.

56. R.H. Loring YS, L. Guo, M. Pires, R. Papke (2013) Biphasic effects of anatabine on nicotinic receptors and NFkB signaling. Society of Neuroscience. San Diego, California, USA: Neuroscience 2013 Abstract

57. Bitner RS, Bunnelle WH, Decker MW, Drescher KU, Kohlhaas KL, et al. (2010) In vivo pharmacological characterization of a novel selective alpha7 neuronal nicotinic acetylcholine receptor agonist ABT-107: preclinical considerations in Alzheimer's disease. J Pharmacol Exp Ther 334: 875-886.

58. Bitner RS, Nikkel AL, Markosyan S, Otte S, Puttfarcken P, et al. (2009) Selective alpha7 nicotinic acetylcholine receptor activation regulates glycogen synthase kinase3beta and decreases tau phosphorylation in vivo. Brain Res 1265: 65-74

59. Del Barrio L, Martin-de-Saavedra MD, Romero A, Parada E, Egea J, et al. (2011) Neurotoxicity induced by okadaic acid in the human neuroblastoma SH-SY5Y line can be differentially prevented by alpha7 and beta2* nicotinic stimulation. Toxicol Sci 123: 193-205.

60. Fionda C, Malgarini G, Soriani A, Zingoni A, Cecere F, et al. (2013) Inhibition of glycogen synthase kinase-3 increases NKG2D ligand MICA expression and sensitivity to NK cell-mediated cytotoxicity in multiple myeloma cells: role of STAT3. J Immunol 190: 6662-6672.

61. Beurel E, Jope RS (2008) Differential regulation of STAT family members by glycogen synthase kinase-3. J Biol Chem 283: 21934-21944. 